\title{
Acquired hemophilia a in a patient undergoing aortic valve replacement with warfarin anticoagulation therapy
}

Keywords: aortic valve replacement, hemophilia A, heparin sodium, prednisolone, warfarin, anti-coagulant

Abbreviations: INR, international normalized ratio; aPTT, activated partial thromboplastin time; F VIII, factor VIII; BU, bethesda units; FFP, fresh frozen plasma; RCC, red cell concentrate

\section{Introduction}

Spontaneous development of acquired hemophilia $\mathrm{A}$ is a rare phenomenon and its origin is usually idiopathic. ${ }^{1}$ However, acquired hemophilia A has been known to occur after surgery ${ }^{2}$ and in underlying diseases such as malignancies and autoimmune diseases. ${ }^{1,3}$ Theodossiades et al. ${ }^{2}$ reported 7 patients with surgery-associated acquired hemophilia ${ }^{2}$ occurring 1-18days after surgery. The surgeries were mainly abdominal surgeries such as cholecystectomy, enteric section and abdominal wall hernia repair. ${ }^{2}$ In contrast, acquired hemophilia A rarely occurs in patients undergoing cardiovascular surgery ${ }^{2}$ or those using the anti-coagulant agent, warfarin..$^{4-5}$ One case of a patient undergoing coronary artery bypass grafting was noted among 7 cases of surgery-associated acquired hemophilia. ${ }^{2}$ A 66-year-old man underwent aortic valve replacement for severe aortic valve stenosis. After surgery, he was administered warfarin $(2 \mathrm{mg} /$ day $)$ for anticoagulation and the international normalized ratio (INR) was controlled between 1.8 and 2.0. Approximately 5months later, he suddenly presented with severe subcutaneous hematoma in the extremities without trauma. Clotting activities worsened (INR, 2.81; activated partial thromboplastin time [aPTT], 103 seconds; normal range, 24-39seconds). Although warfarin was stopped and the INR normalized (INR 1.07), the aPTT remained prolonged (aPTT, 88.9seconds). Other anticoagulants such as heparin sodium and dabigatran were not used. Lupus anticoagulant test (dilute Russel viper venom time) was negative. Therefore, we measured the factor VIII (F VIII) clotting activity and its inhibitor's activity; based on this, the patient was diagnosed with acquired hemophilia A for bleeding tendency (F VIII activity, 1.2\%; F VIII inhibitor, 152 Bethesda units $[\mathrm{BU}])$. From the definite diagnosis, prednisolone $(1 \mathrm{mg} / \mathrm{kg})$ was started. However, active bleeding from the upper gastrointestinal tract suddenly occurred. Massive blood transfusion was given until use of recombinant factor VII therapy. Prior to recombinant factor VII infusion, we administered red cell concentrate (RCC) (26 units) and fresh frozen plasma (FFP) (12units). Two units of RCC $(280 \mathrm{ml})$ or 2 units of FFP $(120 \mathrm{ml})$ were derived from $400 \mathrm{ml}$ of donated whole blood in Japan. After recombinant factor VII infusion, RCC (6units) alone was transfused. Factor VIII inhibitor activity lowered $(<0.5 \mathrm{BU})$ and bleeding tendency resolved. Prednisolone was tapered gradually
Volume 4 Issue 2 - 2017

\author{
Matsunaga $\mathrm{H},{ }^{\prime}$ Mishima $\mathrm{H},{ }^{\prime}$ Katayama $\mathrm{Y}^{\prime}$ ' \\ Ishikawa S,' Hiroshi Fujita ${ }^{2}$ \\ 'Department of Cardiovascular Surgery, Tokyo Metropolitan \\ Bokutoh Hospital, Japan \\ ${ }^{2}$ Department of Transfusion Medicine, Tokyo Metropolitan \\ Bokutoh Hospital, Japan
}

Correspondence: Hiroshi Fujita, Department of Transfusion Medicine, 4-23-I5 Koutoubashi, Sumida-ku, Tokyo I30-8575, Japan, Fax 8|-3-3633-6I73, Tel 8I-3-3633-6I5I, Email hiroshi_fujita@tmhp.jp

Received: October 03, 2016 | Published: March 20, 2017

and then completely discontinued. Fortunately, relapse of acquired hemophilia A was not noted even without prednisolone (aPTT, 26.7$36.0 \mathrm{~s}$ ). Re-administration of warfarin induced good control of the patient's anticoagulation (INR, 1.8-2.0) and no relapse of acquired hemophilia A has been seen.

Three reports have presented5 patients with acquired hemophilia A associated with anticoagulation with warfarin; ${ }^{4-6}$ the 3 case reports are shown in Table 1 . The 5 patients were elderly women (mean age, 68years), while in the present case, the patient was a 66-year-old man. Two among the 5patients were undergoing long-term warfarin therapy for mechanical valve replacement. ${ }^{4-5}$ We speculate that acquired hemophilia may be masked by warfarin therapy among patients undergoing valve replacement. Therefore, prolonged aPTT during warfarin therapy should be noted and checked if it leads to a diagnosis of complicated acquired hemophilia. The 5patients described previously demonstrated a potential delayed diagnosis of acquired hemophilia during anticoagulation with warfarin. The patient in the present case was also administered warfarin for aortic valve replacement, 5 months prior to the onset of hemophilia. He had no history of other diseases, such as malignancy or autoimmune diseases. ${ }^{1}$ Fortunately, he achieved complete remission on prednisolone and no relapse has been noted after discontinuation of prednisolone. Furthermore, readministration of warfarin did not induce relapse, while in previous reports, no data was shown on relapse in 2 patients who took warfarin again. ${ }^{4-6}$ In the present case, because onset of acquired hemophilia occurred approximately 5months after surgery, is it not thought to be surgery-associated. ${ }^{2}$ 
Table I Clinical features of warfarin-masked acquired hemophilia A

\begin{tabular}{|c|c|c|c|c|c|c|c|}
\hline \multirow[b]{2}{*}{$\begin{array}{l}\text { Agel } \\
\text { Gender }\end{array}$} & $\begin{array}{c}\text { Underlining } \\
\text { Diseases }\end{array}$ & \multirow[b]{2}{*}{$\begin{array}{l}\text { Bleeding } \\
\text { Symptom }\end{array}$} & \multirow{2}{*}{$\begin{array}{c}\text { Clotting Tests } \\
\text { aPTT } \\
\text { (s) FVIII } \\
\text { Inhibitor } \\
\text { (Bethesda } \\
\text { units) }\end{array}$} & \multirow[b]{2}{*}{$\begin{array}{l}\text { Hemostatic } \\
\text { Control }\end{array}$} & \multirow[b]{2}{*}{$\begin{array}{l}\text { Immunosuppressive } \\
\text { Treatment }\end{array}$} & \multirow[b]{2}{*}{ Outcome } & \multirow[b]{2}{*}{ Reference } \\
\hline & $\begin{array}{c}\text { Reason of } \\
\text { Warfarin Use }\end{array}$ & & & & & & \\
\hline $72 / F$ & $\begin{array}{c}\text { Mitral valve } \\
\text { replacement (I0 } \\
\text { years ago) } \\
\text { Chronic } \\
\text { Obstructive lung } \\
\text { disease } \\
\text { Diabetes mellitus } \\
\text { Chronic renal } \\
\text { failure } \\
\text { Surgery and } \\
\text { radiation for } \\
\text { Breast cancer (4 } \\
\text { years ago) }\end{array}$ & $\begin{array}{c}\text { Limb hematoma } \\
\text { Hematuria } \\
\text { Pulmonary } \\
\text { hemorrhage }\end{array}$ & $\begin{array}{c}133 \mathrm{~s} \\
4\end{array}$ & $\begin{array}{c}\text { Vitamin K } \\
\text { Intravenous } \\
\text { Immunoglobulin } \\
\text { RCC }\end{array}$ & Prednisolone & Remission & 4 \\
\hline $64 / F$ & $\begin{array}{l}\text { SLE (38 years ago) } \\
\text { Aortic valve } \\
\text { replacement ( } 3 \\
\text { years ago) }\end{array}$ & $\begin{array}{l}\text { Retroperitoneal } \\
\text { hematoma }\end{array}$ & $\begin{array}{c}82 \mathrm{~s} \\
1\end{array}$ & $\begin{array}{c}\text { Vitamin K } \\
\text { RCC, FFP } \\
\text { Desmopresin } \\
\text { Intravenous } \\
\text { Immunoglobulin } \\
\text { Prothrombin } \\
\text { complex } \\
\text { concentrate }\end{array}$ & $\begin{array}{c}\text { Prednisolone } \\
\text { Cyclophosphamide }\end{array}$ & Remission & 5 \\
\hline $60 / F$ & $\begin{array}{c}\text { SLE (20 years ago) } \\
\text { Deep vein } \\
\text { thrombosis }\end{array}$ & Back hematoma & $\begin{array}{c}72 \mathrm{~s} \\
12\end{array}$ & $\begin{array}{l}\text { Vitamin K } \\
\text { RCC, PCC }\end{array}$ & Cyclosporin & $\begin{array}{l}\text { Remission } \\
\text { No relapse } \\
\text { after } \\
\text { reused } \\
\text { warfarin }\end{array}$ & 5 \\
\hline $72 / F$ & $\begin{array}{c}\text { Atrial fibrillation } \\
\text { Deep vein } \\
\text { thrombosis }\end{array}$ & $\begin{array}{c}\text { Retroperitoneal } \\
\text { and inguinal } \\
\text { hematoma }\end{array}$ & $\begin{array}{c}95 \mathrm{~s} \\
2\end{array}$ & $\begin{array}{l}\text { Vitamin K } \\
\text { RCC, FFP } \\
\text { Factor VII } \\
\text { infusion } \\
\text { Prothrombin } \\
\text { complex } \\
\text { concentrate }\end{array}$ & $\begin{array}{c}\text { Prednisolone } \\
\text { Cyclophosphamide }\end{array}$ & Remission & 6 \\
\hline $7 I / F$ & $\begin{array}{c}\text { Systemic sclerosis } \\
\text { Pulmonary } \\
\text { hypertension }\end{array}$ & $\begin{array}{l}\text { Bleeding from } \\
\text { leg ulcer }\end{array}$ & $\begin{array}{l}81 \mathrm{~s} \\
2.2\end{array}$ & $\begin{array}{c}\text { Warfarin } \\
\text { discontinuation }\end{array}$ & None & Remission & 6 \\
\hline $\begin{array}{l}\text { Our case } \\
66 / M\end{array}$ & $\begin{array}{l}\text { Aortic valve } \\
\text { replacement ( } 5 \\
\text { months ago) }\end{array}$ & Limb hematoma & $\begin{array}{l}103 \mathrm{~s} \\
152\end{array}$ & $\begin{array}{l}\text { RCC, FFP } \\
\text { Factor VII } \\
\text { infusion }\end{array}$ & Prednisolone & Remission & \\
\hline
\end{tabular}

\section{Conclusion}

Acquired hemophilia A can be complicated in a warfarinmasked patient undergoing cardiovascular surgery. Immediate and accurate diagnosis of acquired hemophilia A may reduce the usage of transfusion product for bleeding episodes.

\section{Acknowledgements}

None.

\section{Conflict of interest}

The author declares no conflict of interest.

\section{References}

1. Sborov DW, Rodgers GM. How I manage patients with acquired haemophilia A. Br J Haematol. 2013;161(2):157-165.

2. Theodossiades G, Tsevrenis V, Nomikou E, et al. Surgery-associated acquired hemophilia A. Ann Hematol. 2001;80(11):691-693.

3. Makita S, Aoki T, Watarai A, et al. Acquired hemophilia associated with autoimmune bullous diseases: A report of two cases and a review of the literatures. Intern Med. 2013;52(7):807-810.

4. Kantor R, Mayan H, Puritz L, et al. Acquired hemophilia masked by warfarin therapy. Am J Med Sci. 2000;319(3):197-201. 
5. Uggla B, Linder O, Schulman S. Acquired hemophilia masked by warfarin therapy: report on two cases. Blood Coagul Fibrinolysis. 2003;14(8):769-772.
6. Vadikolia CM, Riddell A, Brooks S, et al. Acquired haemophilia masked by warfarin therapy. Int J Lab Hematol. 2007;29(1):64-68. 